\title{
What is a Surgical Complication?
}

\author{
Daniel K. Sokol · James Wilson
}

(C) Société Internationale de Chirurgie 2008

In preparing for a lecture on the ethics of surgical complications, it became apparent that confusion exists about the definition of a "surgical complication." Is it, as one medical website states, "any undesirable result of surgery?" [1]. In the European Journal of Surgery, Veen et al. [2] provide a more elaborate definition: "every unwanted development in the illness of the patient or in the treatment of the patient's illness that occurs in the clinic" [2]. An esteemed historian of science suggests yet another definition in a recent volume on surgical complications: "a complication, in any sphere of endeavour, is something out of the norm, and the product of extraneous and unexpected factors" [3]. Such is the discrepancy in definitions that Rampersaud et al. [4] declared in 2006 that "presently, there is no clear or consistent definition of a complication in the surgical literature."

Much research in surgery aims to reduce the risk of surgical complications. However, until we have a stable and agreed definition of what counts as a surgical complication, we cannot reliably compare different studies to discover what best reduces the chance of surgical complications [5]. Therefore, the topic is more than mere pedantry; defining surgical complications will help us with the broader question of how to improve surgical practice.

A basic PubMed search returned nearly 800 articles with the phrases "surgical complications" or "surgical complication" in the title. But unlike the sources above,

D. K. Sokol ( $\bowtie)$

St George's, University of London, Cranmer Terrace, London SW17 0RE, United Kingdom

e-mail: daniel.sokol@talk21.com

J. Wilson

Centre for Professional Ethics, Keele University, Keele, Staffordshire ST5 5 BG, United Kingdom many articles on the subject do not define surgical complications at all. A complication for one author may not be so for another. In Lewis Carroll's Through the Looking Glass, Humpty Dumpty declares "When I use a word, it means just what I want it to mean" [6]. Mr Dumpty's subjective approach to language is best avoided when dealing with an issue as common and significant as surgical complications.

The website's definition, "any undesirable result of surgery," captures an essential part of a complication: it must be undesirable. There is no such thing as a good surgical complication. The definition, although pleasingly simple, is nonetheless inadequate. An unsightly operative scar is undesirable but not necessarily a complication. As Clavien et al. [7] have noted, a surgical scar is generally considered a sequela, i.e., an adverse outcome inherent to the procedure. To avoid conflating surgical complications with sequelae, we can appeal to the notion of expectation:

1. A surgical complication (SC) is any undesirable and unexpected result of an operation. A scar need not be a complication. This will depend on whether it was expected. Our revised definition, however, does not specify the subject of the unpleasant result, hence a surgeon's needlestick injury would constitute a surgical complication. The solution is to specify the recipient of the SC:

2. A SC is any undesirable and unexpected result of an operation affecting the patient.

Veen et al.'s definition limits complications to what happens in the clinic, but some complications, such as postoperative stroke, develop days after the operation. What matters is not that it occurs in the clinic but that the complication develops as a direct result of the operation, hence: 
3. A SC is any undesirable and unexpected result of an operation affecting the patient that occurs as a direct result of the operation.

The historian's definition, like number 3 above, requires a surgical complication to be unexpected. On reflection, however, this condition is incomplete. Although it may be true that most complications are unexpected, it does not follow that unexpectedness is required. To illustrate, imagine performing an extremely high-risk abdominal aortic aneurysm repair with a $60 \%$ risk of rupture. Imagine further that despite your best efforts the rupture occurs. In this case, it seems plausible to say that, although you expected the rupture, it was still a surgical complication, albeit a likely one. A plausible explanation is that you did not intend to cause the rupture. A surgical complication cannot be intended. Another explanation is that, although the rupture was expected, it was still reasonable to hope for a good outcome. If the risk was $95 \%$, then we would be more reluctant to say that the rupture was a surgical complication. Unlike the $60 \%$ case, it is clearly unreasonable to expect a successful outcome with such odds. To rescue our definition, we need to specify that a SC must be unintended and that there must be a reasonable hope that the operation will succeed. Hence, the full definition reads:

4. A SC is any undesirable, unintended, and direct result of an operation affecting the patient, which would not have occurred had the operation gone as well as could reasonably be hoped.

The revised definition suggests that a surgical complication is not a fixed reality, but that it is dependent on the level of surgical skill and the facilities available. A surgical complication in the United Kingdom may not count as a surgical complication in rural India and similarly a complication in 2005 may not have been in 1808. As surgical techniques and equipment improve, what were once inevitable adverse outcomes may acquire the status of surgical complications. Paradoxically, the better our surgical skill and the higher our expectations, the more potential surgical complications there are.

Any definition that includes the notion of reasonableness raises alarm bells. It is clear that if a procedure has a $1 /$ 1000 chance of success, it would be unreasonable to expect it to succeed and hence such a failure would not count as a surgical complication, at least under our definition. The opposite is true if a procedure has a $9 / 10$ chance of success. Deciding whether an expectation is reasonable will be harder when the likelihood falls between the two extremes of certainty. It will to some extent be a matter of judgment.

A moment's reflection on the definition above raises further issues, which we shall only touch upon. Who, for instance, must judge the result undesirable: the patient, the surgeon, the medical profession? If a procedure somehow gave the patient superhuman strength in his arm, would that be a surgical complication? How do we know whether an adverse event is directly or indirectly caused by an operation?

This brief analysis perhaps explains the reason for the confusion in the literature. There is confusion because defining a surgical complication is no easy task.

The astute reader will have noticed that our definition captures all instances of surgical negligence, as well as nonnegligent complications. Cases of surgical negligence constitute a subset of surgical complications. This raises an important ethical issue around labeling an adverse surgical outcome as a complication. Although strictly speaking true, it would be misleading to describe swabs inadvertently left in the abdomen as a surgical complication. By omitting to specify the nature of the complication, the speaker hopes the hearer will draw a false conclusion, namely that there was no negligence. The philosopher Bernard Williams gives another such example of a true but deceptive utterance. If I open my colleague's mail while he is away on holiday and, upon his return, tell him that "someone has been opening your mail," I am strictly speaking telling the truth but still using unspoken assumptions to fool him [8]. The principle of honesty requires not just the avoidance of lying, but the sharing of relevant and sufficiently detailed information, as determined by social norms and expectations.

The definition provided in this article seems to capture our reflective intuitions on what constitutes a surgical complication. To the best of our knowledge, it is one of the very few detailed examinations of this oft-used term in the medical literature [7,9]. Whether researchers decide to use it as a working definition, it is important that they reveal their chosen definition in their work. Only then will the current veil of confusion be lifted.

Acknowledgments The authors thank Dr. Simisade Adedeji and the Journal's three anonymous reviewers for their help and advice.

\section{References}

1. Website Liposuction.com. Available at: http://www.liposuction. com/safety. Accessed August 28, 2007

2. Veen M, Lardenoye J, Kastelein G, Breslau P (1999) Recording and classification of complications in a surgical practice. Eur $\mathbf{J}$ Surg 165:421-424

3. Chapman A (2007) A history of surgical complications. In: Hakim N, Papalois V (eds) Surgical complications. Imperial College Press, London, pp 1-40

4. Rampersaud Y, Moro E, M-A N et al (2006) Intraoperative adverse events and related postoperative complications in spine surgery: implications for enhancing patient safety founded on evidencebased protocols. Spine 31:1503-1510

5. Clavien P-A, Sanabria J, Strasberg S (1992) Proposed classification of complication of surgery with examples of utility in cholecystectomy. Surgery 111:518-526 
6. Carroll L (1970) The annotated Alice. Penguin, London

7. Clavien P-A, Camargo C, Croxford R, Langer B, Levy G, Greig P (1994) Definition and classification of negative outcomes in solid organ transplantation. Ann Surg 220:109-120

8. Williams B (2002) Truth and truthfulness. Princeton University Press, Princeton
9. Clavien P-A, Sanabria J, Strasberg S (1992) Proposed classification of complications of surgery with examples of utility in cholecystectomy. Surgery 111:518-526 